\title{
0939 EVALUATION THE RISK OF VIOLENCE BETWEEN INDIVIDUALS WITH PARANOID DISORDER
}

I Filov*, M Raleva, D J Peshevska Correspondence:Community Mental Health Center, Ul.Bitolska b.b.Demir Hisar 00389, Macedonia, The Former Yugoslav Republic

10.1136/ip.2010.029215.939

Introduction The connection between psychiatric disorders and aggressive behaviour, especially homicides, as extreme shape of human aggression, is confirmed with many surveys. The purpose of the investigation is to analyse psycho pathological, psychological and social characteristics of subgroups of patients, which have been diagnosed as paranoid states and which is with risk of aggressive manifestation, including homicides.

Material and Methods The investigation was conducted in the Psychiatric Hospital- Demir Hisar and the Community Mental Health Centre-Prilep. Experimental and control group has 50 patients. In the first group, the patients were homicide perpetrators, and in the second group, they were not perpetrators of any criminal act. Both groups were submitted to BPRS (Brief Psychiatric Rating Scale), LOBI (List Of Basic Information), Paranoid Scale, Scale of Aggression (Cattel) MM PI and Forensic Questionnaire.

Conclusions Investigation shows increased values of the most of the variables of the experimental group, compared with the same variables of the control group.

Discussion The specific psycho pathological variables, which are connected with committing homicide, are anxiety, hostility, grandiosity, aggression, uncooperativeness and excitement. The psychological variables are the increase values of the paranoid symptoms and aggression, socio pathological orientation and the increase value of the hysteric scale. They are separated according to the significance in the homicide desire of the persons with paranoid states. The evaluation of these variables in everyday clinical practice of the persons with paranoid states means the base for prevention and prediction of the aggressive behaviour, including homicide. 